\title{
FACTORES DE RIESGO ASOCIADOS A NEUMONÍA NOSOCOMIAL EN PACIENTES ADULTOS
}

\author{
RISK FACTORS ASSOCIATED WITH NOSOCOMIAL PNEUMONIA IN ADULT PATIENTS \\ Gonzalo Huaman - Junco 1 ,a
}

\begin{abstract}
RESUMEN
Objetivo: El objetivo de éste artículo es realizar una revisión sistemática de diversas publicaciones científicas acerca de los factores de riesgo asociados a neumonía nosocomial en pacientes adultos. Métodos: La revisión se realizó a través de la búsqueda electrónica de diversos artículos científicos relacionados con el tema. Se utilizó la pregunta PEO: ¿Existe asociación entre los factores de riesgo y el desarrollo de neumonía nosocomial en pacientes adultos? Las fuentes de búsqueda fueron PubMed y Scielo. Las palabras clave fueron: "nosocomial pneumonia", "hospital - acquired pneumonia" en combinación con "risk factors"; "neumonía nosocomial", "neumonía intrahospitalaria" junto con "factores de riesgo". Se seleccionaron los artículos publicados desde 1 de enero de 2014 hasta 10 de diciembre de 2018. Resultados: De los 101 artículos encontrados se descartó 77 por no cumplir con nuestros criterios de inclusión y exclusión, quedando 24 artículos para ésta revisión. Los factores de riesgo significativos para NN fueron: $\operatorname{Edad}(\mathrm{OR}=1.742, \mathrm{p}=0.000)$, trastorno de conciencia (HR: 2.0, $\mathrm{P}<0$,005), ERC (OR: 2.194; $\mathrm{P}<0.002$ ), EPOC (OR: 3.52; $\mathrm{P}=0.001$ ), intubación traqueal de urgencia (OR 1.511; $\mathrm{P}<0.024)$ y ventilación mecánica (HR: 8.2, P < 0,005), etc. Conclusión: Diversos factores de riesgos intrínsecos y extrínsecos se asocian a la adquisición de neumonía nosocomial en pacientes adultos: Edad, trastorno de conciencia, ERC, EPOC, intubación endotraqueal, ventilación mecánica y otros factores más.
\end{abstract}

Palabras clave: Neumonía nosocomial; Neumonía intrahospitalaria; Factores de riesgo. (fuente: DeCS BIREME)

\begin{abstract}
Objective: The objective of this article is to conduct a systematic review of several scientific publications about the risk factors associated with nosocomial pneumonia in adult patients. Methods: The review was conducted through an electronic search of several scientific articles related to this topic. We used the following PEO question: Is there an association between risk factors and the development of nosocomial pneumonia in adult patients? The search sources were PubMed and Scielo. The keywords used to search were: "nosocomial pneumonia", "hospital - acquired pneumonia" in combination with "risk factors"; "neumonía nosocomial", "neumonía intrahospitalaria" together with "factores de riesgo". Articles published from January 1, 2014 to December 10, 2018 were selected. Results: Of the 101 articles found, 77 were discarded for not meeting our inclusion and exclusion criteria, leaving 24 articles for this review. Significant risk factors for NN were: Age $(O R=1,742, p=0,000)$, consciousness disorder (HR: 2.0, $\mathrm{P}<0.005), \mathrm{CKD}$ (OR: 2,194; $\mathrm{P}<0.002$ ), COPD (OR: 3.52; $\mathrm{p}=0.001$ ), emergency tracheal intubation (OR $1,511 ; \mathrm{P}<0.024)$ and mechanical ventilation (HR: 8.2, $\mathrm{P}<0.005$ ), etc. Conclusion: Several intrinsic and extrinsic risk factors are associated with the acquisition of nosocomial pneumonia in adult patients: age, consciousness disorder, CKD, COPD, endotracheal intubation, mechanical ventilation and other factors.
\end{abstract}

Key words: Nosocomial pneumonia; Hospital - acquired pneumonia; Risk factors. (source: MeSH NLM)

Citar como: Gonzalo Huaman-Junco. Factores de riesgo asociados a neumonía nosocomial en pacientes adultos. [Artículo de Revisión].2019;19(1):80-89. (Enero 2019). DOI 10.25176/RFMH.v19.n1.1796 


\section{INTRODUCCIÓN}

La neumonía nosocomial (NN) es la infección que afecta el parénquima pulmonar (alveólos) que se manifiesta después de 48 horas o más de la admisión del paciente al hospital, evidenciable por un nuevo infiltrado en la radiografía de tórax, y que durante el ingreso no estaba presente ni en periodo de incubación. Además si la neumonía se asocia con una intervención diagnóstica o terapéutica también se considera nosocomial, a pesar que se produzca dentro de éste periodo de 72 horas, por ejemplo después de la intubación endotraqueal. La neumonía asociada a uso de ventilación mecánica (NAV) aparece después de 48 - 72 horas de la realización de intubación endotraqueal en el paciente. Es pertinente destacar que muchos pacientes con neumonía nosocomial son tratados fuera de la unidad de cuidados intensivos (UCl) y no requieren ventilación mecánica) $)^{1-3}$. La NN es una de las infecciones intrahospitalarias más comunes, ocurriendo con una frecuencia de 5 a más de 20 casos por cada 1000 admisiones hospitalarias, y es una de las principales causas de muerte, morbilidad y uso de recursos en pacientes hospitalizados. Además, la mayor cantidad de casos de NN ocurre en pabellones de hospitalización convencional, con una incidencia de 3 a 7 episodios por cada 1000 ingresos ${ }^{1}$. Por otra parte, la distribución de las infecciones en los nosocomios no es homogénea $y$, a pesar que sólo 5 $10 \%$ de los pacientes admitidos a un hospital están en $\mathrm{UCl}, 20-25 \%$ del total de las infecciones adquiridas en el hospital ocurre en estas unidades; siendo la neumonía nosocomial la infección diagnosticada con mayor frecuencia en UCl. Para poder desarrollar estrategias efectivas para prevenir la $\mathrm{NN}$ en la $\mathrm{UCl}$, primero se necesita identificar correctamente los factores que incrementan el riesgo de adquirir la infección ${ }^{4}$. Esto también es relevante para todas las unidades de hospitalización convencionales médicas o quirúrgicas. El objetivo de éste artículo es realizar una revisión de la bibliografía publicada sobre los factores de riesgo asociados a neumonía nosocomial en pacientes adultos.

\section{MÉTODOS}

En este trabajo de revisión sistemática se utilizó artículos en inglés y español publicados desde 1 de enero de 2014 hasta 10 de diciembre de 2018 en PubMed y Scielo. Se utilizó la pregunta PEO: ¿Existe asociación entre los factores de riesgo y el desarrollo de neumonía nosocomial en pacientes adultos? Población: Pacientes adultos Exposición: Factores de riesgo Resultado: Neumonía nosocomial Las palabras clave fueron: "risk factors" (MeSH Term) en combinación con "nosocomial pneumonia" y "hospital - acquired pneumonia" (utilizados en PubMed); "neumonía nosocomial", "neumonía intrahospitalaria", "factores de riesgo" (utilizados en Scielo). El trabajo se encuentra en las prioridades sanitarias "Enfermedades transmisibles: Infecciones intrahospitalarias, Infecciones respiratorias, Neumonía". Según las "Prioridades Nacionales de Investigación en Salud 2015- 2021".

\section{Criterios de inclusión:}

- Artículos publicados en los últimos 5 años. Artículos que incluyan alguna de las siguientes combinaciones de palabras clave en el título o resumen: neumonía nosocomial o neumonía intrahospitalaria junto con factores de riesgo; "nosocomial pneumonia" o "hospital - acquired pneumonia" junto con "risk factors".

\section{Criterios de exclusión:}

- Estudios que incluyan pacientes con neumonía adquirida en la comunidad (NAC). Pacientes pediátricos

\section{- Estudios descriptivos}

- Estudios que no tengan relación directa con nuestro tema.

- Artículos que no consignen autor o Digital Object Identifier System (DOI).

\section{RESULTADOS}

De los 101 artículos encontrados, se descartó 48 artículos por no cumplir alguna de nuestras combinaciones de palabras clave en el resumen, además se retiró: 7 artículos de pacientes pediátricos, 7 artículos que incluían pacientes con neumonía adquirida en la comunidad, 3 estudios descriptivos, 1 artículo sin autor, 1 artículo sin DOI y 11 artículos por no tener relación directa el tema de revisión. En total se descartó 77 artículos por lo tanto quedaron 24 artículos para ésta revisión bibliográfica. De los 24 artículos seleccionados, 21 contaban con resultados de investigación y 3 se consideraron para aspectos teóricos - conceptuales que tienen relación con el objetivo del presente estudio: Factores de riesgo en neumonía nosocomial (NN).

Los factores de riesgo asociados al desarrollo de neumonía en pacientes no ventilados y ventilados tienen algunas similitudes. Así, pueden tener en común la presencia de factores de riesgo intrínsecos, es decir que están relacionados con el propio huésped como las enfermedades crónicas. También pueden estar expuestos a factores de riesgo extrínsecos como el uso de antibióticos que pueden incrementar el riesgo de colonización de la orofaringe; y factores de riesgo que aumenten la probabilidad de aspirar secreciones orofaríngeas en el árbol respiratorio inferior'. En otras palabras, los factores de riesgo extrínsecos son los procedimientos diagnósticos y terapéuticos que aumentan la posibilidad de desarrollar una enfermedad ${ }^{4}$. 


\section{En los estudios prospectivos se encontró lo siguiente:}

El estudio tipo casos y controles de Sopena y colaboradores, tuvo el objetivo de determinar los factores de riesgo asociados y el pronóstico de neumonía nosocomial en salas de hospitalización general. Se incluyó pacientes adultos con edad $\geq 18$ años, hospitalizados entre enero de 2006 y abril de 2008; provenientes de pabellones de medicina interna, hematooncología, cardiología y otras especialidades médicas; además de servicios de cirugía: general, torácica, traumatológica, neurocirugía y otros. Se definió como casos a los pacientes diagnósticados con NN y se asignó aleatoriamente dos controles (pareados por edad, sexo y fecha de admisión) por cada uno. Además se excluyó a los pacientes que adquirieron neumonía en la UCI. El grupo de estudio fue 119 casos y 238 controles. En los resultados se halló que la incidencia de NN en las salas de hospitalización general fue 2.45 casos por cada 1000 ingresos. La distribución de casos de NN fue de $62.2 \%$ en las salas de medicina clínica y $37.8 \%$ en los servicios de cirugía. La media de la edad de los casos fue $70 \pm 14$. 46 años, el $73 \%$ fueron varones. En el análisis multivariado se encontró; que la malnutrición (OR: 3.41, $\mathrm{p}=0.01$ ), la enfermedad renal crónica (OR: 3.14, p =0.008), la anemia (OR: 2.08, $p=0.02$ ), el trastorno de conciencia (OR: 2.14, p = 0.04), el Índice de Comorbilidad de Charlson $\geq 3$ (OR:1.91, $p=0.04$ ) son factores de riesgo intrínsecos independientes asociados a NN; mientras que la admisión al hospital en el mes previo (OR: 3.24, $p=0.008$ ), y la cirugía torácica (OR: 6.03, $p=0.02$ ) son factores de riesgo extrínsecos independientes asociados a NN. Además se determinó que las complicaciones clínicas ocurrieron en el $57.1 \%$ de los casos, las más frecuentes de éstas fueron: insuficiencia respiratoria $(52.9 \%)$, shock séptico $(10.1 \%)$, falla renal aguda (7.6\%). Finalmente, el $32.7 \%$ de pacientes fallecieron y el $27.7 \%$ de éstas muertes fueron atribuidas a neumonía 5 . El estudio de cohortes de GuzmánHerrador B. y colaboradores, tuvo como objetivo identificar los factores intrínsecos y extrínsecos que incrementaban de forma independiente el riesgo de desarrollar NN en la UCI. Se incluyó a todos los pacientes adultos admitidos al menos 24 horas en la $\mathrm{UCl}$ de un hospital español desde enero de 2006 hasta diciembre de 2009. El total de pacientes estudiados fue 4427, de los cuales el $66 \%$ fueron hombres la media de edad fue 58.8 años. La estancia promedio en UCI fue de 8.1 días $(\mathrm{DE}=10.6)$. Además, 233 pacientes desarrollaron NN mientras fueron admitidos a ésta unidad. La incidencia acumulada de pacientes con NN fue de 5.3 por cada 100 admisiones. La duración promedio de estancia en $\mathrm{UCI}$ previa al diagnóstico de NN fue 7.4 días ( $\mathrm{DE}=8.8$ ). En los resultados del análisis multivariado se encontró que el factor que más influenció en la adquisición de NN fue la exposición a ventilación mecánica (HR: 8.2,
$P<0,005)$. El uso de sonda nasogástrica, otro factor extrínseco, también se asoció significativamente con NN (HR: 2.3, P < 0,005). Los factores intrínsecos que se relacionaron de forma significativa con la NN fueron: Disminución del nivel de conciencia en la admisión (HR: 2.0, P < 0,005) y el índice APACHE II (HR: 1.018, P $<0,005)^{4}$.

Ewan y colaboradores, realizaron un estudio de cohortes, para investigar la asociación de la NN con los factores de riesgos odontológicos y microbiológicos. Por lo tanto, se obtuvo una serie de hisopados de garganta y lengua de pacientes con edad $\geq$ de 65 , con fractura de miembro inferior. Se usó la reacción en cadena polimerasa para detectar las bacterias. De los 93 pacientes, 44 tenían patógenos simples $(n=22)$ o mixtos $(n=22)$. De 51 eventos de colonización, la colonización con S. pneumoniae fue la más común, ( $n=27)$, seguido de $\mathrm{H}$. influenzae y otros. Los microorganismos colonizadores se detectaron por primera vez después de 72 horas de la admisión, en el $90 \%$ de los casos. La NN no se asoció con los factores odontológicos. La NN se asoció con ser portador oral previo de E. coli/S. aureus/P. aeruginosa/MRSA ( $p=0,002 ;$ OR: 9,48). La incidencia de la NN en aquellos portadores de microorganismos potencialmente patógenos fue del 35\% (4\% en pacientes sin éstos gérmenes). La NN se asoció con una mayor duración de la estancia hospitalaria con una media de 30 días de exceso. En el análisis univariado la NN fue significativamente asociada con S. aureus/ MRSA/P. aeruginosa/E. coli detectados en el día 5 (OR: 4,39) o 14 día de internamiento (OR: 6.69). La NN se asoció con un mayor índice de Charlson ( $p=0,005$; OR: 1.46; IC del 95\%), haber sido admitido de un hospital $(p=0,048 ; O R: 4.18)$, tener cáncer activo $(p=0,001 ; O R$ $17,11)$ o haber tenido un episodio de aspiración ( $p=$ $0,004$; OR 33,86$)^{6}$.

La investigación realizada por Toledo C. y colaboradores buscó caracterizar los factores de riesgo perioperatorios tempranos para las complicaciones pulmonares postoperatorias (POPC) en los pacientes que se someten a cirugías no cardíacas. Del total POPC el $10 \%$ fue NN, la mayoría de ocurrencia tardía. En el análisis de regresión logística se encontró que una capacidad funcional baja (RR: 4,6; IC del 95\%: 2,1 a 10,0), la cirugía mayor (RR: 3,6; IC del 95\%: 1,2 a 10,7), la inestabilidad hemodinámica preoperatoria (RR: 3,4; IC del 95\%: 1,1-10,6), el alcoholismo (RR: 3,3; IC del 95\%: 1,0-10,7), la cirugía no planificada (RR:2,3; IC del 95\%: 1,0-5,2), la puntuación SOFA (RR: 1,1; IC del 95\%: 1,0$1,2)$ y el aumento de la presión venosa central (RR: 1,1; IC del 95\%:1.0-1.1) fueron predictores independientes de POPC.7 Hinduja A. y colaboradores estudiaron la prevalencia, los factores de riesgo, y resultados de las infecciones nosocomiales en pacientes con hemorragia intracerebral espontánea. La infección nosocomial 
más frecuente en este estudio fue la neumonía (18\%). En el análisis de regresión multivariado, el único predictor significativo de la infección nosocomial fue la hemorragia intraventricular (OR: 5,4; IC del 95\%: $1,2-11,4 ; P=0,02)^{8}$. Verelst $S$. y colaboradores investigaron si el hacinamiento en el departamento de emergencias se asocia de forma independiente con el riesgo de muerte y morbilidad intrahospitalaria. Los resultados obtenidos mediante el análisis multivariado indicaron que el hacinamiento en el departamento de emergencia no se asoció independientemente con la mortalidad ( OR:0,94; 95\%[IC] 0,74-1,19; $p=0,6$ ), pero tuvo tendencia a asociarse con una mayor incidencia de NN (OR 1,24; IC del 95\%: 0,96 a 1,62; $p=0,09)^{9}$.

\section{En los estudios retrospectivos se halló los siguientes resultados:}

Uvizl R. y colaboradores realizaron un estudio donde procesaroninformación de todoslos pacientes mayores de 18 años admitidos consecutivamente a UCI desde 2011 hasta 2015. El objetivo fue evaluar los factores de riesgo contribuyen al desarrollo de NN. Se estudió un total de 2229 pacientes. De los cuales el $65.9 \%$ fueron hombres y el 34\% mujeres. La mediana de edad fue 63 años. La duración promedio de la estancia en UC fue de 5.9 días. La mortalidad total independiente al diagnóstico fue de $24 \%$. Los criterios para NN fueron cumplidos por 310 pacientes (13.9\%). De este grupo el $14.5 \%$ tuvo NN de inicio temprano y el $85.5 \%$ NN de inicio tardío. Los factores de riesgo no modificables que se asociaron significativamente con NN fueron falla orgánica múltiple (OR: 13.733; $\mathrm{P}<0.0001)$, cardiopatía (OR: 2.255; $\mathrm{P}<0.0001)$ y enfermedad renal crónica (OR: 2.194; $P<0.002$ ). Además, los factores de riesgo modificables que se asociaron de forma significativa a NN fueron: intolerancia a la nutrición enteral (OR: 3.055; $\mathrm{P}<0.0001)$, intubación traqueal de urgencia (OR $1.511 ; \mathrm{P}<0.024)$, reintubación (OR 1.851; $\mathrm{P}<0.001)$ y broncoscopía (OR 2.558; $\mathrm{P}<0.0001)^{10}$.

Stenlund M. y colaboradores realizaron un estudio predictivo con el objetivo de determinar la incidencia y los factores de riesgo potenciales para neumonía adquirida en el hospital en un departamento de cirugía de emergencia para pacientes admitidos por abdomen agudo y traumatismo. Se estudió las historias clínicas de 165 pacientes, de los cuales 90 fueron casos y 75 controles pareados por edad. La NN se diagnosticó en $0.9 \%$ de todos los pacientes y hubo una proporción significativamente mayor en varones $(p=0.025)$. Los potenciales factores de riesgo obtenidos mediante regresión logística simple fueron aspiración sospechada o verificada (OR: 23.9, p<0,001) la cual fue aproximadamente 2 veces mayor que la inmovilización (OR:11.2, p<0,001). Asimismo, el EPOC) Asma (OR: 3,7, p =0,05), la cirugía abdominal (OR:3.2, $\mathrm{p}<0,001$ ), el vómito / la retención gástrica (OR: 2.2, $\mathrm{P}$ $=0.012)$, constituyeron factores de riesgo para $\mathrm{NN}^{11}$.
Minakuchi y colaboradores realizaron un estudio de casos y controles, para determinar los factores de riesgo asociados a NN por aspiración en pacientes hemodializados. Se encontró una incidencia de 5,4\% de NN por aspiración. El análisis multivariable reveló que los factores de riesgo para contraer neumonía aspirativa incluyen: edad ( $p=0.0056, \beta=0.267$ ) índice de masa corporal $(p=0.0312, \beta=-0.486)$, niveles de creatinina sérica $(p=0.0021, \beta=-1.050)$, disminución mensual de creatinina ( $p<0.0001, \beta=4.890)$. Además se identificó que la albuminemia( $p=0.0413, \beta=$ $-4.659)$, colesterol total basal $(p=0.0306, \beta=-0.486)$, la tasa de reducción de albumina $(p=0.0055, \beta$ $=18.161)$ y creatinina $(p=0.0280, \beta=211.79)$ y la duración de la neumonía aspirativa ( $p=0.0001, \beta=$ 6.436) fueron factores de riesgo independientes para mortalidad $^{12}$.

Zuo M. y colaboradores también estudiaron factores de riesgo asociados a la neumonía nosocomial en pacientes en hemodiálisis. Se usó un diseño de casos y controles se analizó los datos de pacientes entre enero de 2008 - diciembre de 2015. Los factores de riesgo significativos en el análisis multivariado fueron: la edad del paciente (OR: 2.029, $\mathrm{p}=0.024)$, el tiempo curativo inicial (OR: 1.960, $p=0.005)$, el trastorno de conciencia (OR: 1.616, $\mathrm{p}=0.000)$, las enfermedades subyacentes( OR:10.484, $\mathrm{P}=0.001$ ) la falla orgánica (OR: 2.154, $\mathrm{p}=$ 0.002 ) el índice de comorbilidades de Charlson (OR: $1.278, p=0.008$ ) y el índice APACHE II (OR: 1.186, $p=$ $<0.001)^{13}$. Zhu y colaboradores realizaron un estudio casos y controles con el objetivo de identificar si la fibrilación auricular (FA) es un factor independiente que aumenta el riesgo de NN. De los pacientes con FA el $25.64 \%$ tuvo NN mientras que solo el $3.66 \%$ de los controles la padeció. En el análisis multivariado se halló que la FA es un factor de riesgo independiente para NN (OR $=13.386, p=0.000)$.El incremento en el riesgo también se asoció a hipertensión arterial (OR $=4.695, \mathrm{p}=0.000)$ insuficiencia cardiaca $(\mathrm{OR}=2.854$, $\mathrm{p}=0.000)$ y la edad $(\mathrm{OR}=1.742, \mathrm{p}=0.000)^{14}$.

Evaristo-Méndez $\mathrm{G}$, Rocha-Calderón $\mathrm{CH}$, se realizaron un estudio transversal para definir factores de riesgo de NN en pacientes sometidos a cirugía abdominal. Se reclutó pacientes mayores de 18 años, operados tanto de urgencia y de forma electiva, contando también a los pacientes con patologías oncológicas intraabdominales y de cirugía general. Se excluyó a los pacientes con ventilación mecánica previa a la cirugía, a los operados por vía laparoscópica, pacientes con traumatismo como causa de la cirugía abdominal, pacientes con diagnostico preoperatorio de infección, y a aquellos con reoperaciones en el mismo ingreso. El total de pacientes analizados fue 942 de los cuales el 9.6\% tenía la infección pulmonar. La edad media en el grupo de pacientes con NN fue de $63 \pm 16$ años. En el grupo de cirugía general falleció 
el $9.2 \%$ mientras que el $7.7 \%$ de cirugía oncológica también falleció. La mortalidad total fue $8.8 \%$ la cual fue atribuida directamente a la infección pulmonar. El análisis multivariado identificó que la edad mayor a 60 años (OR: 2.34; $p=0.001$ ), el ser fumador (OR: 9.48; $\mathrm{p}=0.001)$, el EPOC (OR: 3.52; $\mathrm{p}=0.001)$, la cirugía de urgencia (OR: 2.48; $p=0.001$ ), la anestesia general (OR: 3.18; $p=0.423)$, la duración de la cirugía $\geq 120 \mathrm{~min}$ (OR : 5.79; $p=0.001$ ), el tiempo de estancia en la $\mathrm{UCl} \geq$ 7días (OR :1.23; $\mathrm{p}=0.001$ ), la duración de la ventilación mecánica asistida $\geq 4$ días (OR :5.93; $p=0.001$ ) y el tiempo de hospitalización postquirúrgica $\geq 15$ días (OR :1.20; $p=0.001$ ) son factores de riesgo predictivos e independientes que se asocian al desarrollo de NN postquirúrgica ${ }^{15}$.

Kim $\mathrm{T}$ y colaboradores, realizaron un estudio de casos y controles con el objetivo de determiner los factores de riesgo para NN causada por bacterias Gram negativas resistentes a carbapenems (CRGNB), se incluyó pacientes adultos críticamente enfermos provenientes de 8 hospitales de tercer nivel. 82 pacientes con 86 aislamientos de CRGNB fueron los casos, mientras que en el grupo control se recluto 122 pacientes con NN por bacterias Gram negativas sensibles a carbapenems. En el análisis multivariado resultaron significativos $(p<0.05)$ : la edad $\geq 70$ (OR 0,46 ), la diabetes mellitus (OR :2,77), el uso previo de carbapenems (OR: 4,87) y puntuación radiológica $\geq 5$ (OR: 3,63 ) se asociaron de forma independiente con la NN causada por la $\mathrm{CRGNB}^{16}$. En el estudio de Wang Z. y colaboradores se investigó los factores de riesgo relacionados con la NN postoperatoria en pacientes con cáncer de pulmón estadios I - III a. La incidencia de neumonía postoperatoria en estos pacientes con cáncer de pulmón fue del 2,9\% (15 casos). Además, el análisis multivariado reveló que la edad avanzada (>60 años) (OR 5.813, $\mathrm{p}=0.018$ ) y el tipo histopatológico de carcinoma escamoso (OR 5.831, $\mathrm{p}<0.001)$ fueron también factores de riesgo independientes estadísticamente significativos para la neumonía postoperatoria ${ }^{17}$.

El estudio de Divani AA. y colaboradores tuvo el objetivo de identificar los factores de riesgo asociados a NN en pacientes con hemorragia intracerebral espontánea. Se observó $19.6 \%$ de ocurrencia de NN en la población estudiada. Los resultados obtenidos mediante el análisis multivariado indicaron que: la aspiración en el hospital (HR : $3.03, p=0.0003$ ), la intubación (HR: 2.31, $\mathrm{p}=0.01$ ), independientemente de donde se realizó, ya sea fuera del hospital, en el departamento de emergencia o en la neuro - UCl; y la traqueostomía (HR 2.37, p = 0.001). fueron predictores significativos de NN. En los pacientes sobrevivientes la media de hospitalización para los que tenían NN fue de 20 días y en el grupo sin ésta enfermedad fue de 4 días $^{18}$. Li YJ y colaboradores investigaron los factores de riesgo, las características clínicas y los resultados clínicos de la NN causada por una infección con Acinetobacter baumannii extensamente resistente a los medicamentos (XDRAB). La mayoría de las 52 cepas de $A$. baumannii $(\mathrm{N}=37,71,2 \%)$ se recogieron en $\mathrm{UCl}$. Mediante la disco difusión clasificó las cepas aisladas en 17 cepas multirresistentes y 35 extensivamente resistentes .La puntuación APACHE ॥ (OR: 1,17; P : 0,034), EPOC (OR: 7,25; P: 0,012), y las cardiopatías (OR: 6,94; $\mathrm{p:}$ 0,016) fueron identificados como factores de riesgo independientes para la adquisición de $\mathrm{XDRAB}^{19}$.

En el estudio realizado por Liu C y colaboradores se investigó los factores de riesgo asociados con la resistencia al imipenem en pacientes con NN debido a Achromobacter xylosoxidans. El EPOC y la enfermedad arterial coronaria fueron factores de riesgo independientes según el análisis multivariado ${ }^{20}$. El estudio de Dudau D y colaboradores tuvo el objetivo de identificar los factores predictivos tempranos de la recurrencia de la NN después de un trasplante de pulmón o de corazón-pulmón. De los pacientes transplantados el $47 \%$ adquirió NN en la UCI. El $40 \%$ de los pacientes con NN tuvo recurrencia de NN. La gravedad de la enfermedad (puntuación SOFA) y la lesión pulmonar (valor de la puntuación radiológica) fueron los dos factores de riesgos principales de la recurrencia de la $\mathrm{NN}^{21}$. SánchezEncinales $\mathrm{V}$ y colaboradores, investigaron la asociación de la sobreproducción de la proteína A de membrana externa $(\mathrm{OmpA})$ del Acinetobacter baumannii con la neumonía, bacteriemia y mortalidad. El análisis multivariado en las dos cohortes estudiadas mostró que la sobreexpresión de OmpA es un factor de riesgo independiente de neumonía (OR:4.81, $\mathrm{P}<0,001$ ), bacteriemia (OR: 2.41, P : 0,005) y muerte (OR: 1.62 $, \mathrm{P}: 0,049)^{22}$. Buendgens et al. realizaron un estudio retrospectivo en pacientes de $\mathrm{UCl}$ donde encontraron que no había asociación significativa entre los inhibidores de bomba de protones y la NN23. Artículo de revisión de Bardou M et al: Dos metanálisis de ECCA de 2012 y 2013 que compararon fármacos para profilaxis de ulceras de estrés no proporcionaron pruebas de un mayor riesgo de NN atribuible a los IBP. Además, el metanálisis que comparó la profilaxis con el placebo o ninguna profilaxis no indicó un aumento del riesgo de neumonía nosocomial en el grupo de profilaxis (RR 1,16; IC del 95\%: 0,84 a 1,58). Un ECCA realizado de 2010 a 2011 encontró un aumento significativo en la incidencia de neumonía en pacientes que usaban pantoprazol ${ }^{24}$. 
Tabla 1. Títulos, autores y diseño de los trabajos de investigación relacionados a la neumonía nosocomial y sus factores de riesgo.

\begin{tabular}{|c|c|c|c|c|c|}
\hline AUTORES & TÍTULO & AÑo & PAís & POBLACIÓN & DISEÑO \\
\hline $\begin{array}{l}\text { Guzmán-Herrador B, Díaz Molina } \\
\text { C, Farouk Allam M, } \\
\text { Fernández-Crehuet Navajas R. }\end{array}$ & $\begin{array}{l}\text { Independent risk factors associated with } \\
\text { hospital-acquired pneumonia in an adult ICU: } \\
\text { 4-year prospective cohort study in a university } \\
\text { reference hospital }\end{array}$ & 2015 & España & 4427 & Prospectivo, Cohortes \\
\hline $\begin{array}{l}\text { Uvizl R, Kolar M, Herkel T, Vobrova } \\
\text { M, Langova K }\end{array}$ & $\begin{array}{l}\text { Possibilities for modifying risk factors for the } \\
\text { development of hospital-acquired pneumonia } \\
\text { in intensive care patients: results of a } \\
\text { retrospective, observational study }\end{array}$ & 2017 & República Checa & 2229 & $\begin{array}{l}\text { Analítico, Retrospectivo, } \\
\text { Observacional }\end{array}$ \\
\hline $\begin{array}{l}\text { Sopena N, Heras E, Casas I, } \\
\text { Bechini J, Guasch I, Pedro-Botet } \\
\text { ML, et al. }\end{array}$ & $\begin{array}{l}\text { Risk factors for hospital-acquired pneumonia } \\
\text { outside the intensive care unit: A case-control } \\
\text { study }\end{array}$ & 2013 & España & 357 & $\begin{array}{l}\text { Prospectivo, Casos y } \\
\text { controles }\end{array}$ \\
\hline $\begin{array}{l}\text { Stenlund M, Sjödahl R, Pia } \\
\text { Yngman-Uhlin RN. }\end{array}$ & $\begin{array}{l}\text { Incidence and potential risk factors for } \\
\text { hospital-acquired pneumonia in an } \\
\text { emergency department of surgery }\end{array}$ & 2017 & Suecia & 210 & $\begin{array}{l}\text { Retrospectivo, } \\
\text { Casos y controles }\end{array}$ \\
\hline $\begin{array}{l}\text { Evaristo-Méndez G, Rocha- } \\
\text { Calderón CH. }\end{array}$ & $\begin{array}{l}\text { Factores de riesgo para neumonía nosocomial } \\
\text { en pacientes con cirugía abdominal }\end{array}$ & 2015 & México & 942 & Analítico, Transversal \\
\hline $\begin{array}{l}\text { Divani AA, Hevesi M, Pulivarthi S, } \\
\text { Luo X, Souslian F, et al. }\end{array}$ & $\begin{array}{l}\text { Predictors of Nosocomial Pneumonia in } \\
\text { Intracerebral } \\
\text { Hemorrhage Patients: A Multi-center } \\
\text { Observational Study }\end{array}$ & 2014 & E.E.U.U. & 591 & Analítico, Retrospectivo \\
\hline $\begin{array}{l}\text { Ewan VC, Sails AD, Walls AW, } \\
\text { Rushton S, Newton JL }\end{array}$ & $\begin{array}{l}\text { Dental and Microbiological Risk Factors } \\
\text { for Hospital-Acquired Pneumonia in Non- } \\
\text { Ventilated Older Patients }\end{array}$ & 2015 & Inglaterra & 90 & $\begin{array}{l}\text { Analítico, Prospectivo, } \\
\text { Cohortes }\end{array}$ \\
\hline $\begin{array}{l}\text { Minakuchi H, Wakino S, Hayashi } \\
\mathrm{K} \text {, Inamoto H, Itoh H. }\end{array}$ & $\begin{array}{l}\text { Serum Creatinine and Albumin Decline } \\
\text { Predict the Contraction of Nosocomial } \\
\text { Aspiration Pneumonia in Patients Undergoing } \\
\text { Hemodialysis }\end{array}$ & 2014 & Japón & 335 & Analítico, Retrospectivo \\
\hline $\begin{array}{l}\text { Zuo M, Tang J, Xiang M, Long } \\
\text { Q, Dai J }\end{array}$ & $\begin{array}{l}\text { Characteristics and factors associated with } \\
\text { nosocomial pneumonia among patients } \\
\text { undergoing continuous renal replacement } \\
\text { therapy (CRRT): A case-control study }\end{array}$ & 2018 & China & 1160 & $\begin{array}{l}\text { Retrospectivo, Casos y } \\
\text { controles }\end{array}$ \\
\hline Zhu J, Zhang X, Shi G, Yi K, Tan X. & $\begin{array}{l}\text { Atrial Fibrillation Is an Independent Risk } \\
\text { Factor for Hospital-Acquired Pneumonia }\end{array}$ & 2015 & China & 8657 & $\begin{array}{l}\text { Retrospectivo, Casos y } \\
\text { controles }\end{array}$ \\
\hline $\begin{array}{l}\text { Wang Z, Cai XJ, Shi L, Li FY, Lin } \\
\text { NM. }\end{array}$ & $\begin{array}{l}\text { Risk Factors of Postoperative Nosocomial } \\
\text { Pneumonia in Stage I - Illa Lung Cancer } \\
\text { Patients }\end{array}$ & 2014 & China & 511 & Analítico, Retrospectivo \\
\hline $\begin{array}{l}\text { Sánchez-Encinales V, Álvarez- } \\
\text { Marín R, Pachón-lbáñez ME, } \\
\text { Fernández-Cuenca F, Pascual } \\
\text { A, et al. }\end{array}$ & $\begin{array}{l}\text { Overproduction of Outer Membrane Protein } \\
\text { A by Acinetobacter baumannii as a Risk Factor } \\
\text { for Nosocomial Pneumonia, Bacteremia, and } \\
\text { Mortality Rate Increase. }\end{array}$ & 2017 & España & 346 & $\begin{array}{l}\text { Post hoc analysis of } \\
\text { unicenter cohort and a } \\
\text { multicenter cohort }\end{array}$ \\
\hline $\begin{array}{l}\text { Kim T, Chong YP, Park SY, Jeon } \\
\text { MH, Choo EJ, et al. }\end{array}$ & $\begin{array}{l}\text { Risk factors for hospital-acquired pneumonia } \\
\text { caused by carbapenem-resistant Gram- } \\
\text { negative bacteria in critically ill patients: a } \\
\text { multicenter study in Korea }\end{array}$ & 2014 & Korea & 320 & $\begin{array}{l}\text { Retrospectivo, Casos y } \\
\text { controles, Multicéntrico }\end{array}$ \\
\hline $\begin{array}{l}\text { Li YJ, Pan CZ, Fang CQ, Zhao ZX, } \\
\text { Chen HL }\end{array}$ & $\begin{array}{l}\text { Pneumonia caused by extensive drugresistant } \\
\text { Acinetobacter baumannii among hospitalized } \\
\text { patients: genetic relationships, } \\
\text { risk factors and mortality }\end{array}$ & 2017 & China & 52 & $\begin{array}{l}\text { Retrospectivo, Casos y } \\
\text { controles }\end{array}$ \\
\hline $\begin{array}{l}\text { Liu C, Guo J, Yan W, Jin Y, Pan } \\
\text { F,et al. }\end{array}$ & $\begin{array}{l}\text { Hospital-acquired pneumonia due to } \\
\text { Achromobacter xylosoxidans in the elderly: A } \\
\text { single-center retrospective study in Beijing }\end{array}$ & 2017 & China & 41 & $\begin{array}{l}\text { Retrospectivo, Casos y } \\
\text { controles }\end{array}$ \\
\hline $\begin{array}{l}\text { Dudau D, Camous J, Marchand } \\
\text { S, Pilorge C, Rézaiguia-Delclaux } \\
\text { S, et al. }\end{array}$ & $\begin{array}{l}\text { Incidence of nosocomial pneumonia and risk } \\
\text { of recurrence after antimicrobial therapy in } \\
\text { critically ill lung and heart-lung transplant } \\
\text { patients }\end{array}$ & 2014 & Francia & 79 & Analítico, Retrospectivo \\
\hline $\begin{array}{l}\text { Toledo C, Nácul FE, Knibel MF, } \\
\text { Silva NB, Rezende E, et al. }\end{array}$ & $\begin{array}{l}\text { Pulmonary complications after non-cardiac } \\
\text { surgeries: temporal patterns and risk factors }\end{array}$ & 2017 & Brazil & 885 & $\begin{array}{l}\text { Prospectivo, Cohortes, } \\
\text { Multicéntrico }\end{array}$ \\
\hline $\begin{array}{l}\text { Toledo C, Nácul FE, Knibel MF, } \\
\text { Silva NB, Rezende E, et al. }\end{array}$ & $\begin{array}{l}\text { Nosocomial infections in patients with } \\
\text { spontaneous intracerebral hemorrhage }\end{array}$ & 2015 & E.E.U.U. & 202 & Analítico, Prospectivo \\
\hline $\begin{array}{l}\text { Verelst } \mathrm{S} \text {, Wouters } \mathrm{P} \text {, Gillet JB1, } \\
\text { Van den Berghe G. }\end{array}$ & $\begin{array}{l}\text { Emergency department crowding in relation } \\
\text { to in-hospital adverse medical events: a large } \\
\text { prospective observational cohort study }\end{array}$ & 2015 & Bélgica & 108229 & Prospectivo, Cohortes \\
\hline
\end{tabular}


Tabla 2. Importancia, fortalezas y debilidades de los trabajos de investigación relacionados a la neumonía nosocomial y sus factores de riesgo.

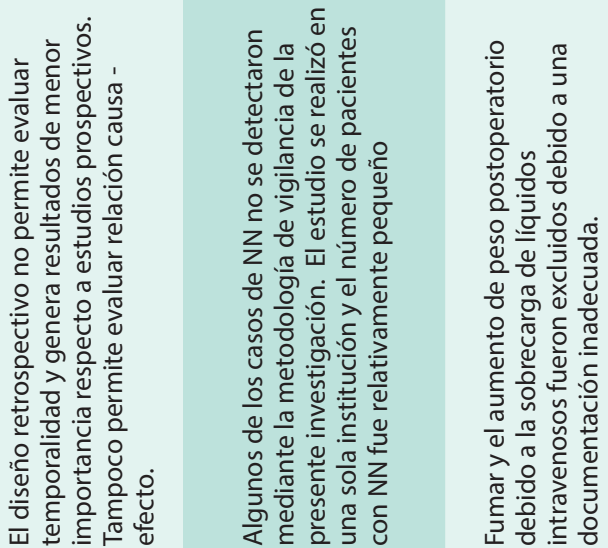

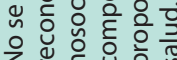

3
0
0
2
0
0
0
0
0
0
0
0

준

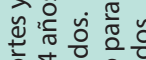

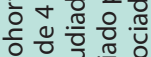

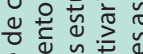

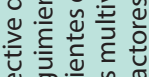

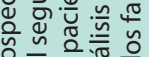

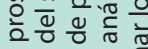

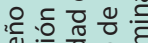

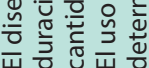

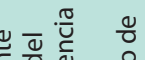

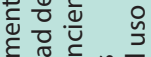

每

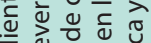

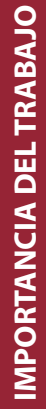

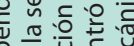

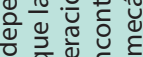

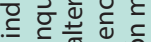

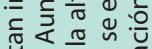

跑

है

告

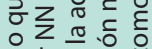

它

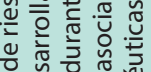

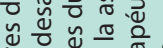

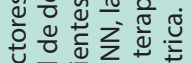

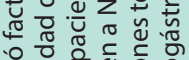

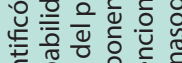

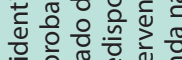

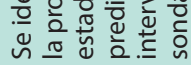

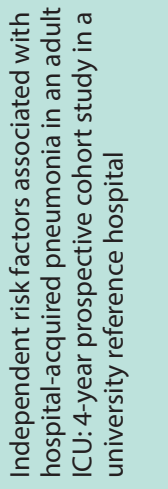

응

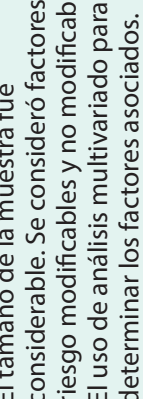

증

产

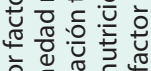

흔

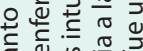

过

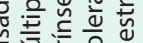

헌

๑)

บ

중

夜

$\sum_{0}$

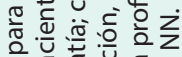

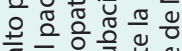

एक

ह 웜

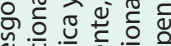

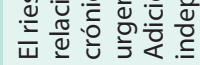

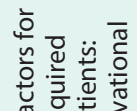

离

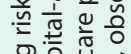

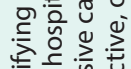

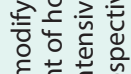

흥

运

票

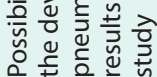

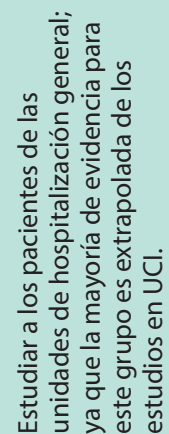

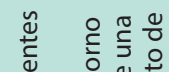

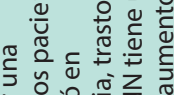

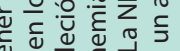

年

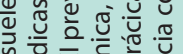

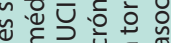

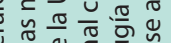

눙

जू

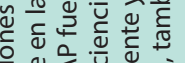

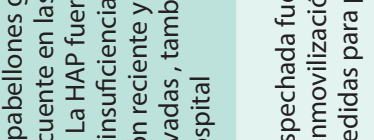

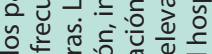

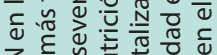

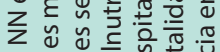

중

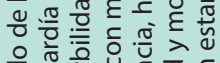

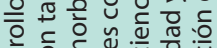

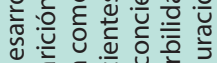

立 品

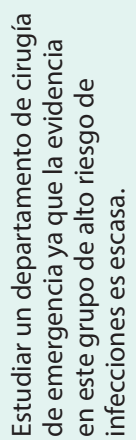

\author{
苋离
}

है है

웡

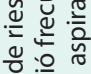

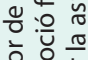

원

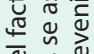

ब.

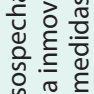



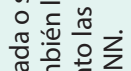

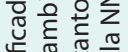

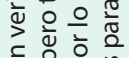

: 응

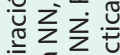

흘

先

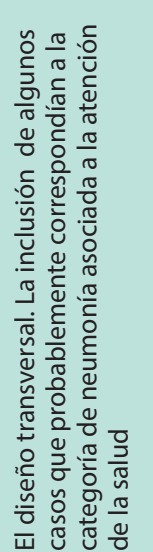

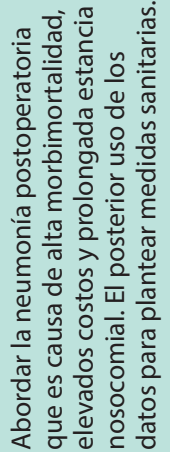

ช)

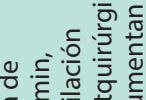

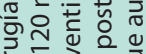

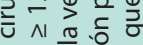

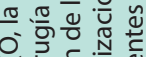

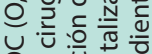

응 는

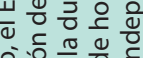

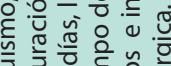

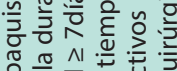

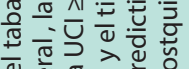

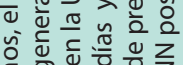

के

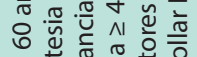

万人

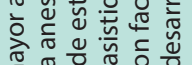

ब.

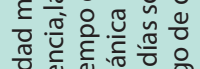

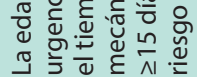

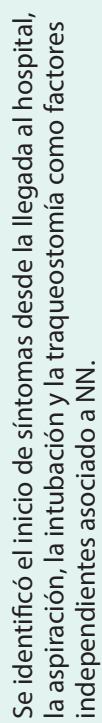

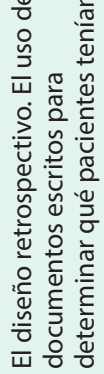

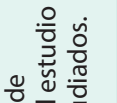

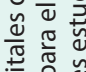

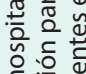

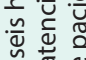

焉

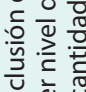

纯

$\checkmark+\lambda$

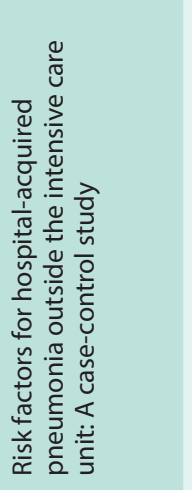

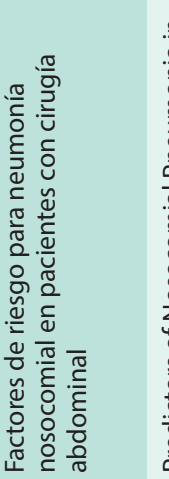

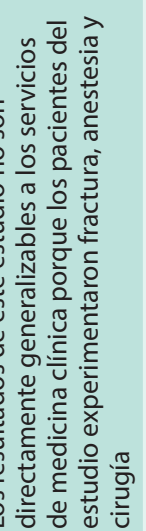

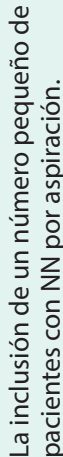

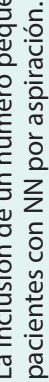

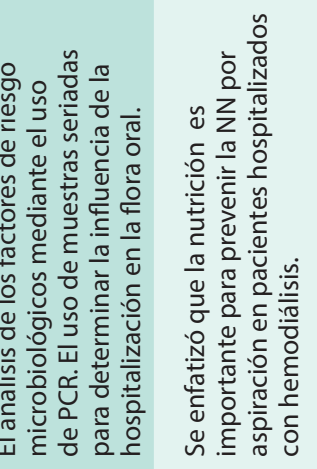

ธิ

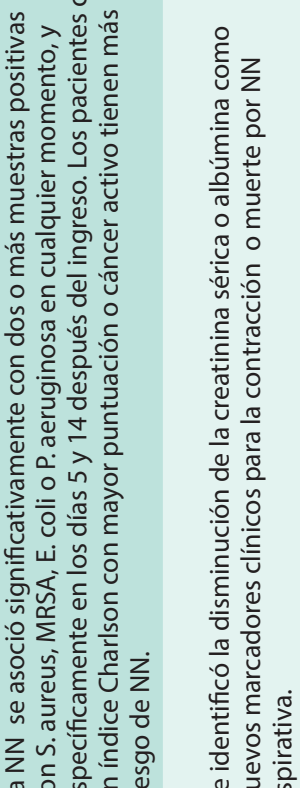

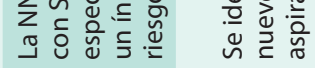

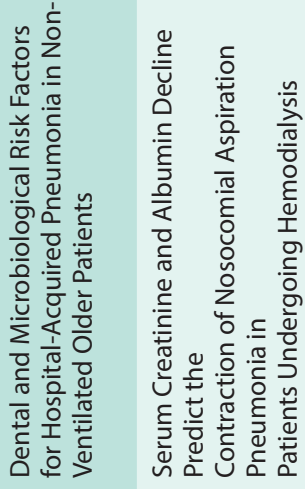




\begin{tabular}{|c|c|c|c|c|c|c|c|c|c|c|}
\hline 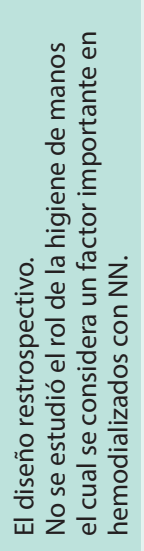 & 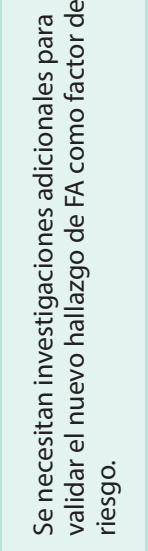 & 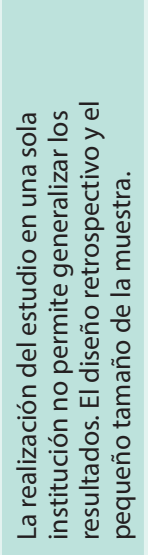 & 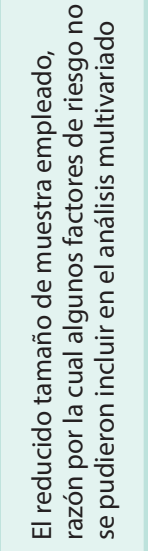 & 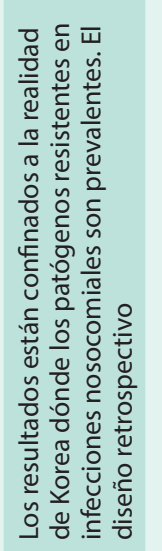 & 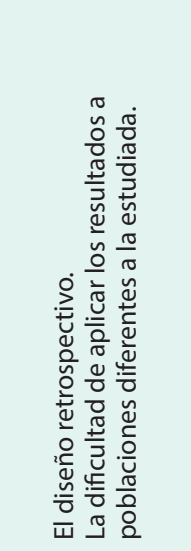 & 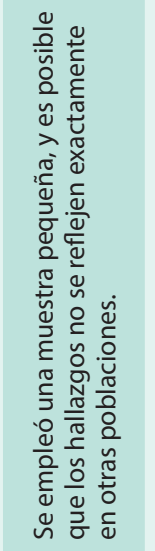 & 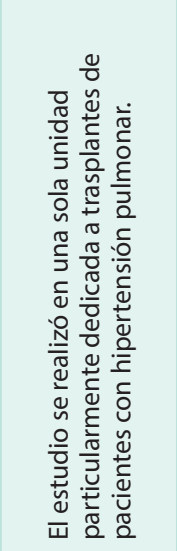 & 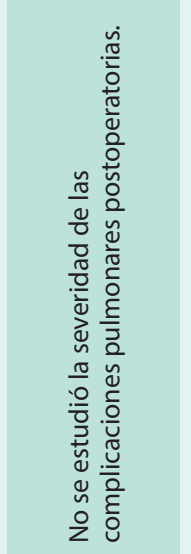 & 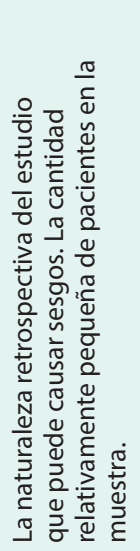 & 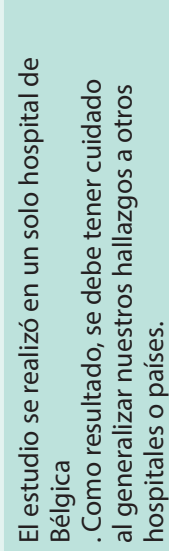 \\
\hline 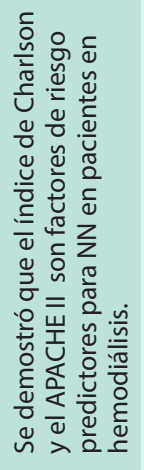 & 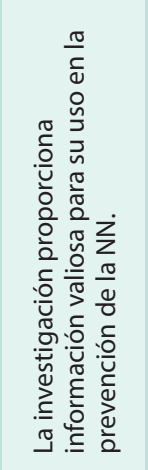 & 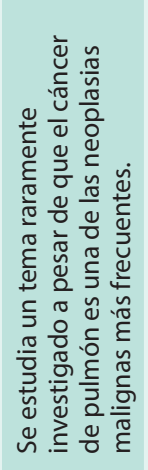 & 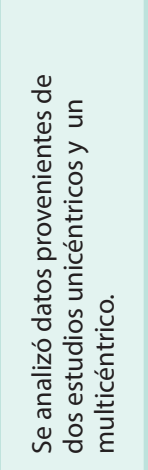 & 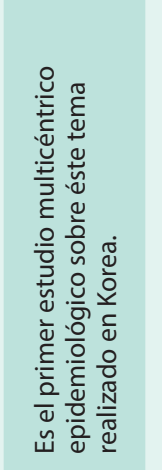 & 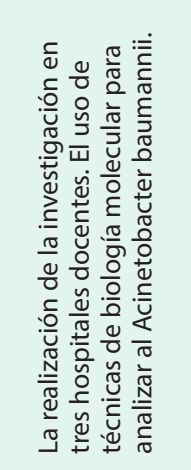 & 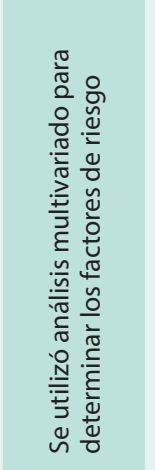 & 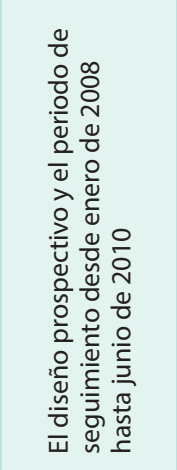 & 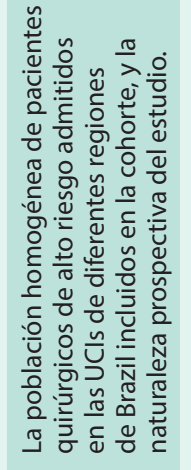 & 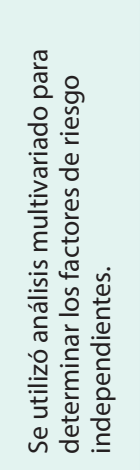 & 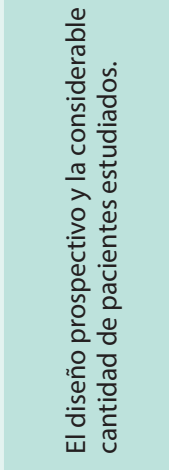 \\
\hline 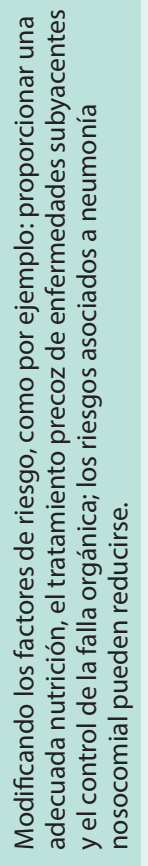 & 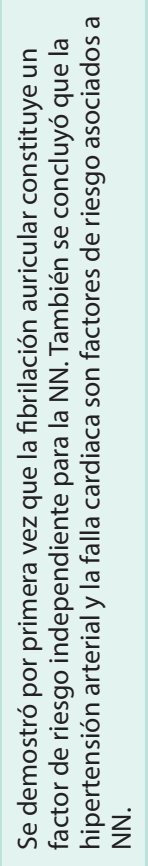 & 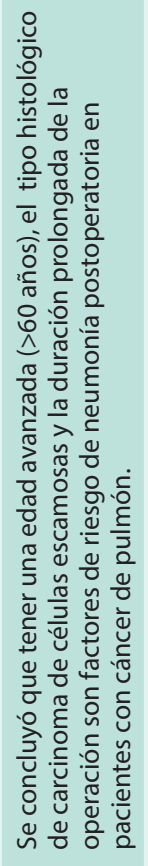 & 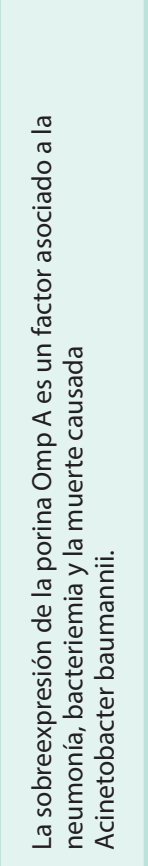 & 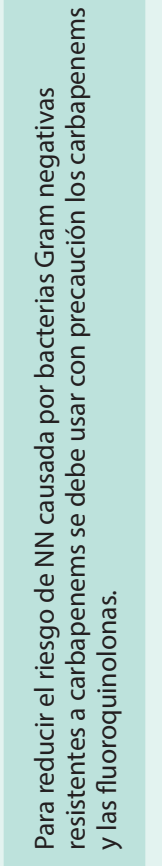 & 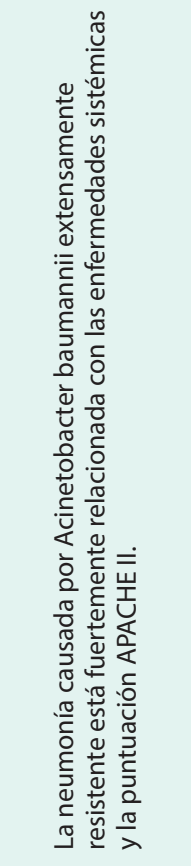 & 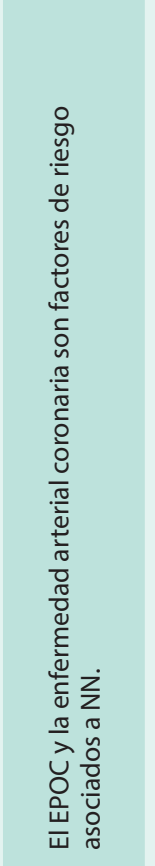 & 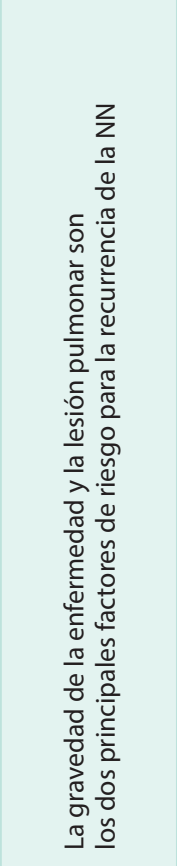 & 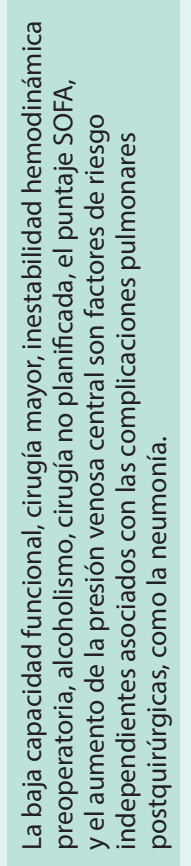 & 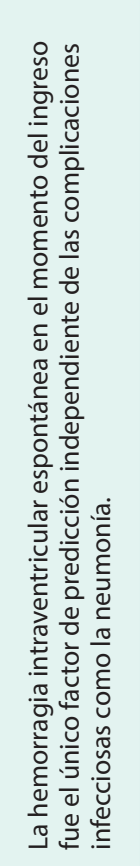 & 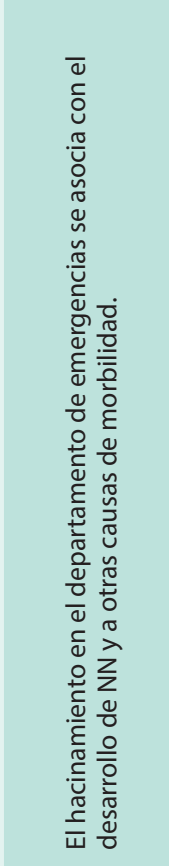 \\
\hline 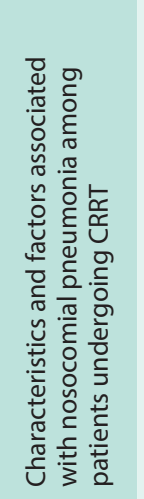 & 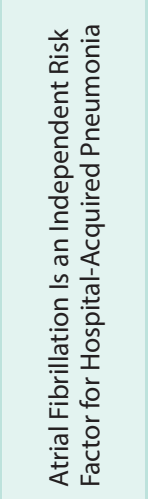 & 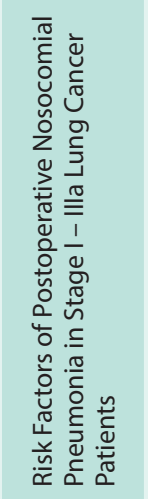 & 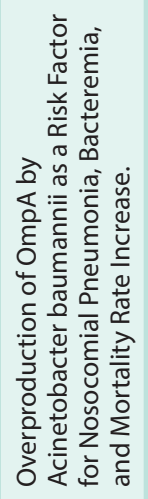 & 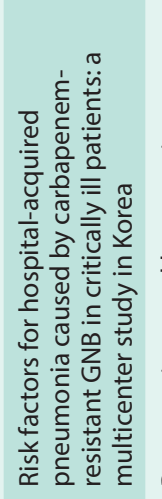 & 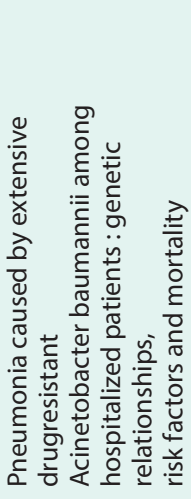 & 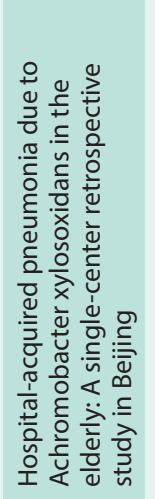 & 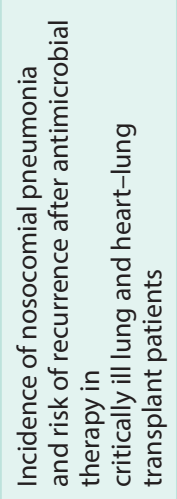 & 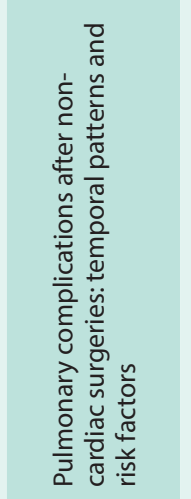 & 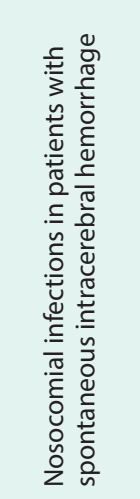 & 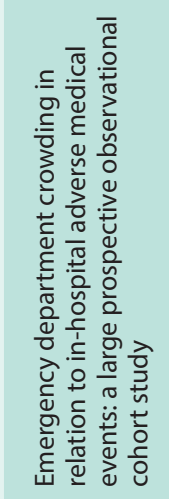 \\
\hline
\end{tabular}




\section{DISCUSIÓN}

Mediante la presente revisión sistemática se observó que hay factores de riesgo para NN que fueron demostrados por varios autores. Además, diversos artículos hallaron factores de riesgo diferentes, ya sea porque no todos los artículos coincidieron en las variables estudiadas o porque éstas no resultaron significativas en el análisis estadístico. Esto puede explicarse por la heterogeneidad entre los estudios respecto a: tipo de población (pacientes de $\mathrm{UCl}$, de cirugía, de medicina clínica, con neoplasias, hemodializados) diseños (prospectivo, retrospectivo), países (de Europa, Asia, América), tipos de bacterias estudiadas y tamaño de la muestra. Los factores de riesgo significativos que coincidieron en más cantidad de estudios fueron en su mayoría intrínsecos al paciente y por tanto con menor potencial de modificarse para prevenir el desarrollo de NN. Los factores de riesgo destacados por la presente revisión, considerando la cantidad de estudios y el nivel de evidencia son: La edad, que fue un factor de riesgo para NN en 6 de 2112-17 estudios, los motivos por los cuales el envejecimiento favorecería la NN podrían explicarse porque la alteración cognitiva, la demencia y el delirio (que son frecuentes en este grupo etario) favorecen la micro o macroaspiración de secreciones orofaringeas que contienen las bacterias patógenas de $\mathrm{NN}^{3}$. Además, la función inmunitaria sufre un deterioro funcional que está asociado con las enfermedades en los ancianos ${ }^{13}$. El trastorno de conciencia, factor asociado en 3 de 21 estudios ${ }^{4,5,13}$ (2 prospectivos), también aumenta la probabilidad de aspiración de microorganismos de la orofaringe ${ }^{4}$ y genera incapacidad de limpiar mecánicamente las secreciones orales ocasionando mayor colonización por bacterias nosocomiales ${ }^{3}$. La enfermedad renal

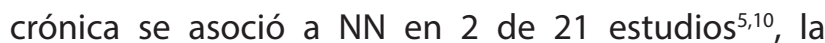
afectación inmunológica que se ve en sus estadios más avanzados predispone a infecciones; además la anemia que aumenta el riesgo de $\mathrm{NN}^{5}$, se presenta con más frecuencia en la ERC. La enfermedad pulmonar obstructiva crónica fue un factor asociado a NN en 4 de 21 estudios ${ }^{11,15,19,20}$, sin embargo dos de estos estudios fueron sobre gérmenes específicos. El score APACHE II fue un factor de riesgo en 3 de 21 estudios $4,13,19$, éste favorecería la NN porque es una medida de la severidad subyacente de la enfermedad ${ }^{4}$. Por lo tanto se requiere más estudios de la utilidad de ésta escala respecto a la adquisición de NN. Respecto a los factores de riesgo extrínsecos la ventilación mecánica (en 2 estudios) $)^{4,15}$ y la intubación endotraqueal (en 2 estudios) $)^{10,18}$ demostraron ser factores de riesgo de NN. La presencia de una vía aérea artificial como el tubo endotraqueal es reconocido como el principal factor de riesgo para microaspiraciones, porque elimina los mecanismos normales de expulsión de secreciones del tracto respiratorio. Además las guías internacionales de prevención recomiendan reducir la cantidad de días en ventilación mecánica y el uso de ventilación no invasiva 4 . Una limitación de la presente revisión es no haber hallado estudios nacionales, lo que podría generar un sesgo.

\section{CONCLUSIÓN}

Diversos factores de riesgo intrínsecos y extrínsecos, potencialmente modificables o no, que se asocian a la adquisición de neumonía nosocomial en pacientes adultos: Edad, trastorno de conciencia, ERC, EPOC, el tabaquismo, intubación endotraqueal, ventilación mecánica, broncoscopía, aspiración, sonda nasogástrica, intolerancia a la nutrición enteral, score APACHE II, índice de Charlson, cardiopatías, fibrilación auricular hipertensión arterial, inmovilización, anemia, malnutrición, falla orgánica múltiple, cirugía mayor, duración de la cirugía, hospitalización previa y otros factores más. Aún se requiere más estudios, en especial multicéntricos y nacionales para caracterizar mejor los factores de riesgo de NN en adultos.

Contribuciones de autoría: El autor Realizó la generación, recolección de información, redacción y aprobación final del artículo original.

Financiamiento: Autofinanciado.

Conflicto de interés: El autor declara no tener conflicto de interés en la publicación de este artículo.

Recibido: 18 de diciembre del 2018

Aprobado: 03 de enero del 2019

Correspondencia: Gonzalo Huamán Junco

Dirección: Jr. El parque 346, San Roque, Surco

Celular: 965410054

Correo:g.hj777@hotmail.com 


\section{REFERENCIAS BIBLIOGRÁFICAS}

1. Díaz E, Martín-Loeches I, Vallés J. [Nosocomial pneumonia]. Enferm Infecc Microbiol Clin. diciembre de 2013;31(10):692-8.

2. Montravers P, Harpan A, Guivarch E. Current and Future Considerations for the Treatment of Hospital-Acquired Pneumonia. Adv Ther. febrero de 2016;33(2):151-66.

3. Ewan V, Hellyer T, Newton J, Simpson J. New horizons in hospital acquired pneumonia in older people. Age Ageing. 1 de mayo de 2017;46(3):352-8

4. Guzmán-Herrador B, Molina CD, Allam MF, Navajas RF-C. Independent risk factors associated with hospital-acquired pneumonia in an adult ICU: 4-year prospective cohort study in a university reference hospital. J Public Health (Oxf). 2016;38(2):378-83.

5. Sopena N, Heras E, Casas I, Bechini J, Guasch I, Pedro-Botet ML, et al. Risk factors for hospital-acquired pneumonia outside the intensive care unit: a case-control study. Am J Infect Control. enero de 2014;42(1):38-42.

6. Ewan VC, Sails AD, Walls AWG, Rushton S, Newton JL. Dental and microbiological risk factors for hospital-acquired pneumonia in nonventilated older patients. PLOS ONE. 2015;10(4):e0123622.

Crit Care. mayo de 2015;24(3):227-31.

9. Verelst S, Wouters P, Gillet J-B, Van den Berghe G. Emergency Department Crowding in Relation to In-hospital Adverse Medical Events: A Large Prospective Observational Cohort Study. J Emerg Med. diciembre de 2015;49(6):949-61.

10. Uvizl R, Kolar M, Herkel T, Vobrova M, Langova K. Possibilities for modifying risk factors for the development of hospital-acquired pneumonia in intensive care patients: results of a retrospective, observational study. Biomed Pap Med Fac Univ Palacky Olomouc Czech Repub. septiembre de 2017;161(3):303-9.

11. Stenlund M, Sjödahl R, Yngman-Uhlin P. Incidence and potential risk factors for hospital-acquired pneumonia in an emergency department of surgery. International Journal for Quality in Health Care. 1 de abril de 2017;29(2):290-4

12. Minakuchi $\mathrm{H}$, Wakino S, Hayashi $\mathrm{K}$, Inamoto $\mathrm{H}$, Itoh H. Serum creatinine and albumin decline predict the contraction of nosocomial aspiration pneumonia in patients undergoing hemodialysis. Ther Apher Dial. agosto de 2014;18(4):326-33.

13. Zuo M, Tang J, Xiang M, Long Q, Dai J, Hu X. Characteristics and factors associated with nosocomial pneumonia among patients undergoing continuous renal replacement therapy (CRRT): A case-control study. Int $J$ Infect Dis. marzo de 2018;68:115-21.

14. Zhu J, Zhang X, Shi G, Yi K, Tan X. Atrial Fibrillation Is an Independent Risk Factor for Hospital-Acquired Pneumonia. PLoS ONE. 2015;10(7):e0131782.

15. Evaristo-Méndez G, Rocha-Calderón $\mathrm{CH}$. Factores de riesgo para neumonía nosocomial en pacientes con cirugía abdominal. Cirugía y Cirujanos. 1 de enero de 2016;84(1):21-7.

16. Kim T, Chong YP, Park SY, Jeon M-H, Choo EJ, Chung J-W, et al. Risk factors for hospital-acquired pneumonia caused by carbapenemresistant Gram-negative bacteria in critically ill patients: a multicenter study in Korea. Diagn Microbiol Infect Dis. abril de 2014;78(4):457-61.

17. Wang Z, Cai X-J, Shi L, Li F-Y, Lin N-M. Risk factors of postoperative nosocomial pneumonia in stage I-Illa lung cancer patients. Asian Pac J Cancer Prev. 2014;15(7):3071-4.

18. Divani AA, Hevesi M, Pulivarthi S, Luo X, Souslian F, Suarez Jl, et al. Predictors of nosocomial pneumonia in intracerebral hemorrhage patients: a multi-center observational study. Neurocrit Care. abril de 2015;22(2):234-42.

19. Li YJ, Pan CZ, Fang CQ, Zhao ZX, Chen HL, Guo PH, et al. Pneumonia caused by extensive drug-resistant Acinetobacter baumannii among hospitalized patients: genetic relationships, risk factors and mortality. BMC Infect Dis. 30 de 2017;17(1):371.

20. Liu C, Guo J, Yan W, Jin Y, Pan F, Fang X, et al. Hospital-acquired pneumonia due to Achromobacter xylosoxidans in the elderly: A singlecenter retrospective study in Beijing. J Infect Dev Ctries. 30 de enero de 2017;11(1):10-8

21. Dudau D, Camous J, Marchand S, Pilorge C, Rézaiguia-Delclaux $S$, Libert J-M, et al. Incidence of nosocomial pneumonia and risk of recurrence after antimicrobial therapy in critically ill lung and heart-lung transplant patients. Clin Transplant. enero de 2014;28(1):27-36.

22. Sánchez-Encinales V, Álvarez-Marín R, Pachón-Ibáñez ME, FernándezCuenca F, Pascual A, Garnacho-Montero J, et al. Overproduction of Outer Membrane Protein A by Acinetobacter baumannii as a Risk Factor for Nosocomial Pneumonia, Bacteremia, and Mortality Rate Increase. J Infect Dis. 15 de 2017;215(6):966-74.

23. Buendgens $L$, Bruensing J, Matthes M, Dückers $H$, Luedde T, Trautwein C, et al. Administration of proton pump inhibitors in critically ill medical patients is associated with increased risk of developing Clostridium difficile-associated diarrhea. J Crit Care. agosto de 2014;29(4):696.e11-15.

24. Bardou M, Quenot J-P, Barkun A. Stress-related mucosal disease in the critically ill patient. Nat Rev Gastroenterol Hepatol. febrero de 2015;12(2):98-107. 